\title{
Vortex fluidic-mediated fabrication of fast gelated silica hydrogels with embedded laccase nanoflowers for real-time biosensing under flow
}

\author{
Xuan Luo, ${ }^{1 *}$ Ahmed Hussein Mohammed Al-Antaki, ${ }^{1}$ Aghil Igder, ${ }^{1,2}$ Keith A. Stubbs, ${ }^{3}$ Peng Su, ${ }^{4}$ Wei \\ Zhang, ${ }^{4}$ Gregory A. Weiss, ${ }^{5}$ Colin L. Raston ${ }^{1 *}$ \\ ${ }^{1}$ Flinders Institute for Nanoscale Science and Technology, College of Science and Engineering, Flinders University, Ade- \\ laide, SA 5042, Australia \\ ${ }^{2}$ School of Engineering, Edith Cowan University, Joondalup, Perth, WA 6027, Australia \\ ${ }^{3}$ School of Molecular Sciences, The University of Western Australia, Crawley, WA, 6009, Australia \\ ${ }^{4}$ Centre for Marine Bioproducts Development, Flinders University, Adelaide, South Australia 5042, Australia \\ ${ }^{5}$ Department of Chemistry, University of California Irvine, CA, 92697-2025, USA
}

KEYWORDS: nanoflowers, enzyme, laccase, biosensor, microfluidics

\begin{abstract}
The fabrication of hybrid protein- $\mathrm{Cu}_{3}\left(\mathrm{PO}_{4}\right)_{2}$ nanoflowers (NFs) via an intermediate toroidal structure is dramatically accelerated under shear using a vortex fluidic device (VFD) which possesses a rapidly rotating angled tube. As-prepared laccase NFs (LNFs) exhibit $\approx 1.8$-fold increase in catalytic activity compared to free laccase under diffusion control, which is further enhanced by $\approx 2.9$-fold for the catalysis under shear in the VFD. A new LNF immobilization platform (VLIS) was subsequently developed by mixing the LNFs for 15 min with silica hydrogel resulting in gelation along the VFD tube surface. The resultant LNFs@Silica coating is highly stable and reusable, which allows a dramatic 16-fold enhancement in catalytic rates relative to LNF@Silica inside glass vials. Real-time monitoring of the UV-Vis within the LNFs@Silica coated tube reveals good stability of the coating in continuous flow processing. The results demonstrate the utility of the VFD microfluidic platform, further highlighting its ability in controlling chemical and enzymatic processes.
\end{abstract}

\section{INTRODUCTION}

The development of enzyme-based biosensors is hindered by the cost and limited reusability of the enzyme. Most enzymes are inactivated during the assay, and cannot be separated from the solution for reuse. Accordingly, there is increasing interest in immobilizing enzymes for use in biosensor applications. Laccase, a glycoprotein with four copper atoms, has significant biotechnological applications towards chemical synthesis, bioremediation, ${ }^{1}$ biobleaching, ${ }^{2}$ and biosensing. ${ }^{3}$ Such commercial potential has attracted attention towards producing high quality stable immobilized laccase. In 2012, Ge et al. reported that copper ions and laccase form a new type of hybrid material, termed $\mathrm{Cu}_{3}\left(\mathrm{PO}_{4}\right)_{2}$ nanoflowers (NFs). ${ }^{4}$ The protein molecules therein form complexes with the $\mathrm{Cu}^{2+}$ ions that become nucleation sites for crystal growth of the flower-like NFs. NFs have significantly enhanced enzymatic activity and excellent thermal stability which allows for high-temperature catalytic reactions to be realised. ${ }^{5}$ However, the structural features of NFs, notably the shape of the petals and dimensions of the flowers are difficult to control during the fabrication, and the synthesis can require three days. ${ }^{6}$ In addition, due to the fragile micron size of NFs, these materials currently require isolation by centrifugation for recycling use, as an energy penalty process, and the multiple centrifugation processing requirements can lead to loss of their structural integrity. ${ }^{7}$

Immobilization of NFs within hydrogel networks offers further protection of enzymes from prod- 
ucts. For example, hybrid NFs entrapped in an agarose hydrogel provide a proficient solid sensing platform for acetylcholine detection by visual inspection. ${ }^{8}$ However, formation of such materials involves time-consuming multi-step reactions, requiring blending premade hybrid NFs (12 h) with un-gelated agarose. An alternative to these methods is in the use of silica-based materials with uniform pore size, tuneable pore network and large surface area, which are gaining interest as supports for immobilizing enzymes. ${ }^{9}$ Laccase catalytic activity, stability and storage capacity ${ }^{10}$ can also be enhanced by immobilizing it with solgel silica. ${ }^{11}$ However, improving sol-gel laccase catalytic activity requires tedious optimisation and relatively time-consuming gelation processes. $^{12}$

We have focused on utilizing thin film microfluidics to mediate protein folding, ${ }^{13}$ biocatalysis ${ }^{14}$ and protein polymerisation, ${ }^{15}$ using the vortex fluidic device (VFD) which confines reagents to a 200 $\mu \mathrm{m}$ film. Importantly, the VFD has been used for effective sol-gel synthesis of silica xerogel from hydrolysis of alkoxysilanes (TMOS) followed by room temperature condensation ${ }^{16}$ with the VFD significantly shortening the gelation time while allowing effective in situ materials incorporation. Here, we wished to establish a new silica-based, enzyme immobilization platform, using the VFD which has intense micromixing and shear stress, as its major modes of facilitating reaction outcomes which are highly amenable to NF formation. We found that the mechanoenergy within the VFD facilitates the interaction between both bovine serum albumin (BSA) or laccase and the $\mathrm{Cu}^{2+}$ ions, leading to an efficient growth of hybrid NFs, BNFs or LNFs, respectively, within $30 \mathrm{~min}$ at $\mathrm{pH} 7.4$ and $25^{\circ} \mathrm{C}$. In addition, the VFD coupled LNF@Silica immobilization system, termed VLIS, is formed in a simple process with the processing complete in 1 h. Each VLIS is reusable, with appreciable Brunauer-Emmett-Teller (BET) surface area and pore volume, and offers rapid, qualitative molecular monitoring in real-time when coupled with in-house designed optical fiber-based probes. Thus, the multi-applicable VFD enables the generation and immobilization of NFs into silica hy- drogels, which greatly simplifies fabrication and offers potential for biosensing applications.

\section{EXPERIMENTAL SECTION}

Bovine serum albumin (BSA), laccase from Trametes versicolor (Sigma 38429), phosphatebuffered saline (PBS), $\mathrm{CuSO}_{4} \cdot 5 \mathrm{H}_{2} \mathrm{O}$, tetramethyl orthosilicate (TMOS) and syringaldazine were purchased from Sigma Aldrich and used without further purification. All stock solutions were prepared with deionized water (MilliQ). $\mathrm{CuSO}_{4}$ solutions was prepared at a concentration of 120 $\mathrm{mM}$ for all reactions with the indicated volumes. For a typical VFD reaction, $10 \mu \mathrm{L}$ of $120 \mathrm{mM}$ $\mathrm{CuSO}_{4}$ was premixed with $1.5 \mathrm{~mL}$ of $0.1 \mathrm{mg} / \mathrm{mL}$ BSA dissolved in $10 \mathrm{mM}$ PBS. Then, $1 \mathrm{~mL}$ of the mixture was quickly transferred into the VFD housing a rotating quartz tube $(20 \mathrm{~mm}$ o.d., 17.5 $\mathrm{mm}$ i.d., length $19.4 \mathrm{~cm}$ ) inclined at $45^{\circ}$ relative to the horizontal position (Figure 1a) for $30 \mathrm{~min}$ of confined mode processing at room temperature. To probe the growth mechanism in the VFD, different reaction times (2, 15 and $30 \mathrm{~min})$ were performed. Control experiments did not use the VFD, and were incubated at room temperature for the specified times. The experiments were repeated three times for reproducibility. The size and morphology of the NFs were examined using a scanning electron microscope (SEM, FEI F50) equipped with energy dispersive X-ray spectroscopy (EDX) capability. XRD patterns were obtained using an X-ray diffractometer (Bruker, Germany) using Co-K $\alpha$ radiation $(\lambda=$ $1.78892 \AA$ ) with the $2 \theta$ scanning range from $10^{\circ}$ to $90^{\circ}$. BET was used to measure the porosity. The elemental and chemical composition was evaluated using Fourier transform infrared spectroscopy analysis (FTIR, Perkin Elmer). Perkin Elmer STA 8000 was used to evaluate the thermal stability of the samples under nitrogen/air atmosphere. The whole heating process were performed with the same ramping rate of $10^{\circ} \mathrm{C} / \mathrm{min}$, under a nitrogen atmosphere.

For determining the encapsulation efficiency, the solution post-VFD processing was centrifuged at $6900 \mathrm{x} g$ for $10 \mathrm{~min}$ to form a pellet, and the protein concentration in the supernatant was analysed by Bradford protein assay (Bio-Rad) following the manufacturer's protocol. Briefly, the standard curve was prepared from a series of PBS 
solutions containing $0,1.25,2.5,510,20$, or 40 $\mu \mathrm{g} / \mathrm{mL}$ BSA followed by the addition of the dye reagent concentrate. After $15 \mathrm{~min}$, the absorbance at $595 \mathrm{~nm}$ was measured. The amount of encapsulated protein was calculated by subtracting the amount of free protein in the supernatant from the total protein used for the synthesis. The encapsulation efficiency was calculated by dividing the amount of encapsulated protein by the total protein used for the reaction. All measurements were performed in triplicate with the average values of three independent experiments reported as mean \pm SEM.

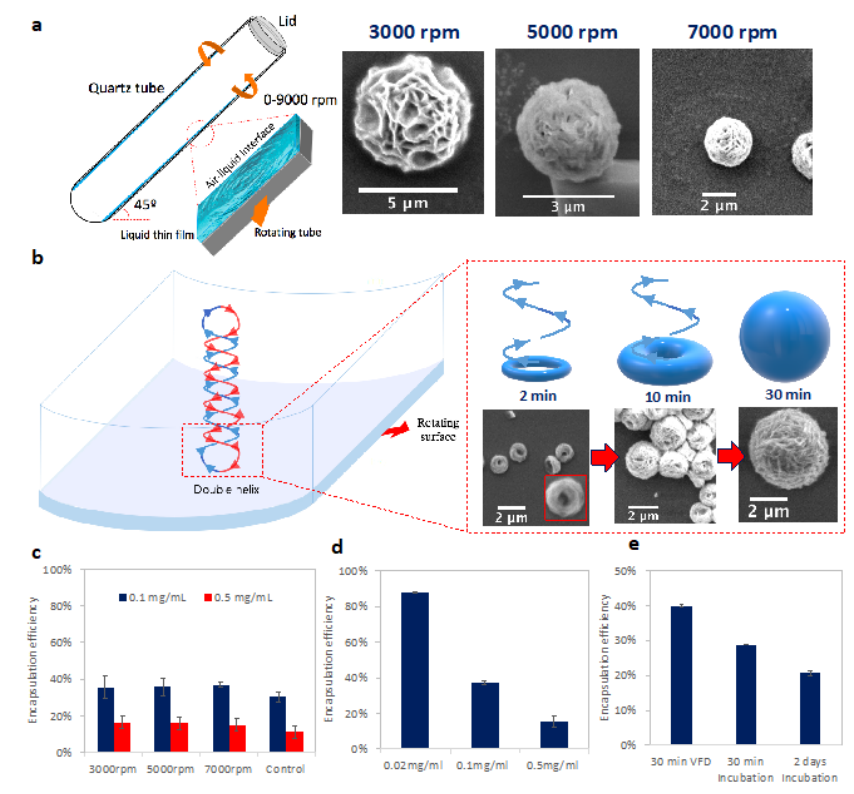

Figure 1: (a) Schematic of a VFD (20 mm o.d. $17.5 \mathrm{~mm}$ i.d. length $19.4 \mathrm{~cm}$ ) operated in confined mode with a tilt angle of $45^{\circ}$. Effect of rotational speed, $\omega$, on the diameter of BNFs after 30 min VFD processing. (b) Formation of BNFs during 2 min (toroidal), 10 min (toroidal with smaller central void) and 30 min (spherical). (c) Encapsulation efficiency of BSA on BNFs at different $\omega$. Experiments were conducted with $10 \mu \mathrm{L}$ of $120 \mathrm{mM}$ $\mathrm{CuSO}_{4}$ premixed with $1.5 \mathrm{~mL}$ of 0.1 or $0.5 \mathrm{mg} / \mathrm{mL}$ BSA dissolved in $10 \mathrm{mM}$ PBS. $1 \mathrm{~mL}$ of the mixture was quickly transferred into the VFD tube for $30 \mathrm{~min}$ processing at $5000 \mathrm{rpm}$, and thereafter the products were centrifuged at $6900 \mathrm{x}$ g for $10 \mathrm{~min}$, and the supernatant used for the protein quantification. (d) Encapsualtion efficiency of BSA with the indicated concentrations of initial BSA. (e) Encapsulation efficiency of BSA after VFD processing of $5000 \mathrm{rpm}$ for $30 \mathrm{~min}$ relative to $30 \mathrm{~min}$ or 2 days incubation.

For stability tests, a suspension of BSA nanoflower (BNFs) was incubated with 25 $\mu \mathrm{g} / \mathrm{mL}$ of trypsin at $25^{\circ} \mathrm{C}$ for $10 \mathrm{~min}$. The BNFs were also challenged by treating with glutaraldehyde $(0.8 \%$, w/w) solution for $48 \mathrm{~h}$, followed by incubation with EDTA (1\%, w/w) as described before. ${ }^{4}$ For calcination, a suspension of BNFs was dried on a silicon wafer and heated at $350{ }^{\circ} \mathrm{C}$ for $2 \mathrm{~h}^{6}{ }^{6}$ For in situ gelation experiments, $10 \mu \mathrm{L}$ of $120 \mathrm{mM} \mathrm{CuSO}_{4}$ solution was premixed with $1.5 \mathrm{~mL}$ of $0.1 \mathrm{mg} / \mathrm{mL}$ BSA dissolved in $10 \mathrm{mM}$ PBS. Then, $1 \mathrm{~mL}$ of the mixture was quickly transferred into the VFD tube for $30 \mathrm{~min}$ processing. Post-VFD processing, $495 \mu \mathrm{L}$ TMOS was added into the VFD tube with the high shear generated at $5000 \mathrm{rpm}$ facilitating mass transfer between the two immiscible liquids, with gelation time reduced to $15 \mathrm{~min}$ in the presence of phosphate. A similar procedure also applies to laccase where $6.7 \mu \mathrm{L}$ of $120 \mathrm{mM} \mathrm{CuSO}_{4}$ with $1 \mathrm{~mL}$ of $0.1 \mathrm{mg} / \mathrm{mL}$ laccase in $20 \mathrm{mM}$ PBS was mixed in VFD at $5000 \mathrm{rpm}$ for $30 \mathrm{~min}$, followed by adding $495 \mu \mathrm{L}$ TMOS for another 15 min VFD processing at $5000 \mathrm{rpm}$ resulting in gelation in situ. When laccase concentration increases $(107.2 \mu \mathrm{L}$ of $120 \mathrm{mM} \mathrm{CuSO}_{4}$ with $1 \mathrm{~mL}$ of $2.5 \mathrm{mg} / \mathrm{mL}$ laccase in $10 \mathrm{mM}$ PBS), LNFs were centrifuged at $6900 \mathrm{x}$ g for $10 \mathrm{~min}$ to a pellet in order to remove the non-encapsulated free laccase. The pellet was then redispersed in $1 \mathrm{~mL}$ of fresh $10 \mathrm{mM}$ PBS followed by adding $495 \mu \mathrm{L}$ TMOS for another 15 min VFD processing at $5000 \mathrm{rpm}$ resulting in gelation in situ. Catalytic activity was evaluated based on the oxidation of syringaldazine at 0.22 $\mathrm{mM}$ between free laccase, LNFs and VFD incorporated LNFs@Silica, as indicated. For the substrate solution, $10 \mathrm{mg}$ of syringaldazine was dissolved in methanol $(50 \mathrm{~mL})$ to generate a stock solution $(0.56 \mathrm{mM})$ and stored wrapped in aluminum foil at $4{ }^{\circ} \mathrm{C}$. Immediately before use, the working concentration of syringaldazine $(0.22$ $\mathrm{mM}$ ) was prepared by adding water. Each reaction was prepared by combining $0.9 \mathrm{~mL}$ laccase solution or PBS and $0.1 \mathrm{~mL}$ of syringaldazine. This solution ( $1 \mathrm{~mL}$ ) was added to either a quartz cuvette or a quartz VFD tube. The reaction was incubated at $25{ }^{\circ} \mathrm{C}$ for 6 min unless otherwise indicated. After the VFD reaction, the sample was immediately transferred to a quartz cuvette and the absorbance was measured at $530 \mathrm{~nm}\left(\lambda_{\max }\right)$. To test the stability of the VFD incorporated LNFs@Silica coating, processing was performed under continuous flow mode where liquids are constantly fed into the bottom of the VFD tube, exiting at the top. The absorbance was measured in real-time with a USB spectrophotometer (Ocean optics, USA) mounted around the tube 
and connected via fibre optics, using OceanView software. This allowed for real-time acquisition and analysis of the reaction.

\section{RESULTS AND DISCUSSION}

BNFs were formed by adding $\mathrm{CuSO}_{4}$ to $\mathrm{PBS}$ containing BSA ( $\mathrm{pH} 7.4)$ at room temperature, Figure 1. SEM images were recorded as a function of the BNFs growth time to ascertain the mechanism and growth pathway. This established the following pathway of formation: toroidal structures, then toroidal structures with the cavity filled, and finally spheroidal particles, Figure $1 \mathrm{~b}$ and Figure S1. In the VFD, the formation of the spheroidal particles is rapid, in contrast to most non-VFD reports in the literature. ${ }^{4}$ Toroidalshaped BSA-Cu ${ }^{2+}$ crystals $(1.5 \mu \mathrm{m}$ in diameter with $0.58 \mu \mathrm{m}$ void diameter) prevail after only 2 min with their formation representing localised areas of high shear associated with double helical topological fluid flow arising from eddies from Faraday waves being twisted by the Coriolis inertial forces from the cylindrical shape of the tube. ${ }^{17}$ They occur across the rotational landscape in the VFD when the tilt angle is $45^{\circ}$, but with reduced dimensionality for increasing rotational speed, ${ }^{17}$ as shown in Figure 1a. The shear stress associated with this double helical fluid flow presumably facilitates protein molecule complexation with $\mathrm{Cu}^{2+}$, noting that the shear stress generated in the VFD is effective in promoting nucleation and growth of nanoparticles. ${ }^{17}$ Complexation predominantly involves coordination of the amide groups in the protein backbone ${ }^{4}$ and the nucleation of $\mathrm{Cu}_{3}\left(\mathrm{PO}_{4}\right)_{2}$ crystals forming stacked petal layers radiating from the centre, thereby filling the void. Processing for $10 \mathrm{~min}$ in the VFD affords slightly larger toroidal structures, with a buildup of material around the surface of the first formed micro-toroids with the void space reducing in size (to $0.25 \mu \mathrm{m}$ in diameter) with the overall diameter of the toroid structures becoming about $2 \mu \mathrm{m}$ (Figure 1b). The growth process continues for longer time, with $30 \mathrm{~min}$ of processing at $45^{\circ}$ resulting in the complete formation of spheroidal BNFs with an increase in the diameter to about $4 \mu \mathrm{m}$. Compared to the control experiment not involving VFD processing or processing at different tilt angles, well-developed
BNFs are rarely observed after 30 min (Figure S2e and Figure S3).

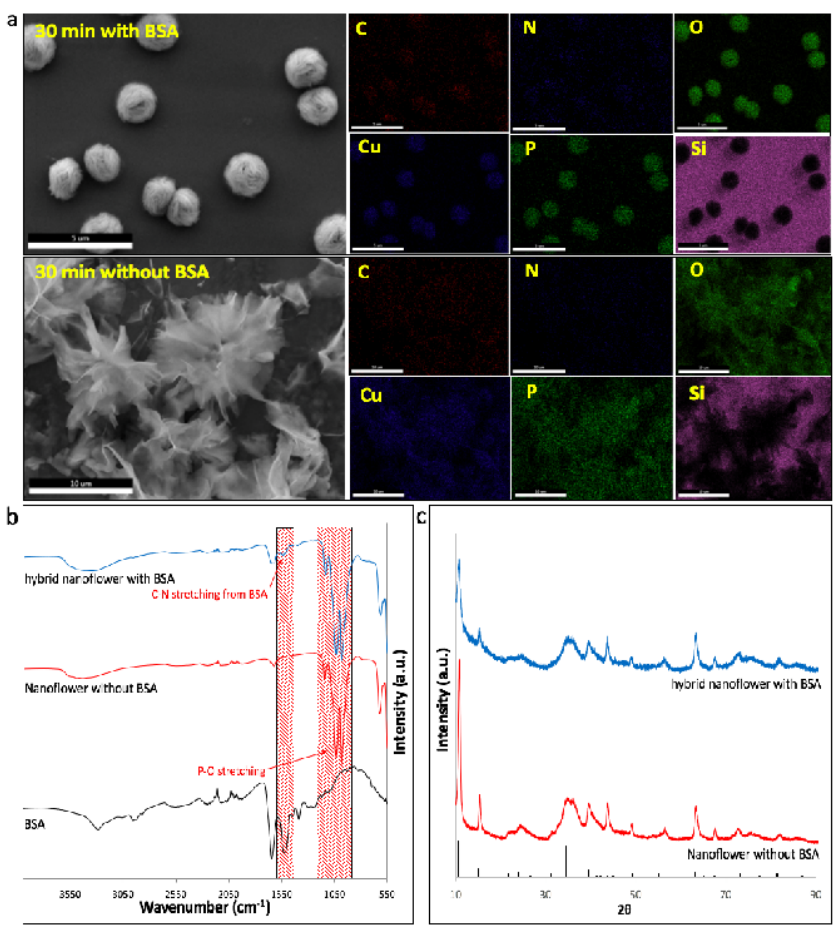

Figure 2. (a) Formation of BNFs with and without BSA using VFD processing at $5000 \mathrm{rpm}$ for $30 \mathrm{~min}$. SEM/EDX images of the corresponding NFs with elemental mapping images for $\mathrm{C}, \mathrm{N}, \mathrm{O}$, $\mathrm{Cu}, \mathrm{P}$ and Si. (b) FT-IR spectra of the BNFs with and without BSA and BSA only. (c) XRD patterns of NFs with and without BSA.

In contrast to conventionally made BNFs where the structures collapse with scattered petals after calcination, trypsin digestion ${ }^{4}$ and glutaraldehyde/EDTA treatment, VFD-generated hybrid NFs have a robust backbone toroidal structure which survives these treatments, with the trypsin treated VFD-prepared toroids not showing significant shrinking or collapsing (Figure S4-5). To confirm the formation of toroidal structures after 2 min of processing, samples were treated with glutaraldehyde (which crosslinks the protein molecules), then incubated with EDTA (to remove $\mathrm{Cu}^{2+}$ ) as described previously. ${ }^{4}$ This results in toroids with significantly smaller sizes $(0.73$ $\mu \mathrm{m}$ in diameter and $0.27 \mu \mathrm{m}$ void diameter) and relatively smooth surfaces, consistent with the protein molecules being located within particles across the toroids (Figure S3). Calcination of both toroids and BNFs at $350{ }^{\circ} \mathrm{C}$ retained the original structures, but the removal of BSA resulted in petals with defects (Figure S3-4). These observations indicate that the growth mechanism of BNFs, and the intermediate toroidal structures, 
within the VFD is dramatically different from those formed using conventional batch processing. The formation of the NFs is initiated from a toroid driven by the double helical flow striking the surface of the VFD tube where there is high heat and mass transfer, ${ }^{17}$ with the protein molecules uniformly distributed within the confines of the toroids.

For 30 min processing, which results in spheroidal BNFs, the size of the BNFs can be manipulated by changing the rotational speed of the VFD (Figure 1a and Figure S2). As shown, BNFs reduced from $5 \mu \mathrm{m}$ to $2 \mu \mathrm{m}$ with more tightly packed petals by increasing the rotational speed from $3000 \mathrm{rpm}$ to $7000 \mathrm{rpm}(1.5 \mathrm{~mL}$ BSA in 10 $\mathrm{mM}$ PBS at $0.1 \mathrm{mg} / \mathrm{mL} ; 10 \mu \mathrm{CuSO}_{4}$ ), in accordance with a reduction in the dimension of the double helical topological flow arising from eddies from the Faraday waves being twisted. ${ }^{17} \mathrm{By}$ systematically exploring the concentration effects (Figure S6) and a wide range of different molar ratios between PBS and $\mathrm{CuSO}_{4}$, we established that $13: 1(1.5 \mathrm{~mL} 0.1 \mathrm{mg} / \mathrm{mL}$ BSA in $10 \mathrm{mM}$ PBS; $10 \mu \mathrm{L} \mathrm{CuSO}_{4}$ ) generates the most homogeneous and monodispersed BNFs (Figure S7-8). On the basis of the encapsulation efficiency for the BNFs prepared under different conditions, the concentration of proteins in the system has greater impact on the encapsulation efficiency (EE) than the rotational speed, with the best outcome (88\% EE) observed when adding a smaller amount of protein, $0.02 \mathrm{mg} / \mathrm{mL}$ for BSA (Figure 1c, d), which is consistent with previous findings. ${ }^{4}$ VFD processsing improves the encapsulation efficiency (EE) of BSA for a processing time of $30 \mathrm{~min}$ by $11 \%$ relative to samples incubated over the same timeframe, and $19 \%$ relative to 2 days incubated samples (Figure $1 \mathrm{e})$.

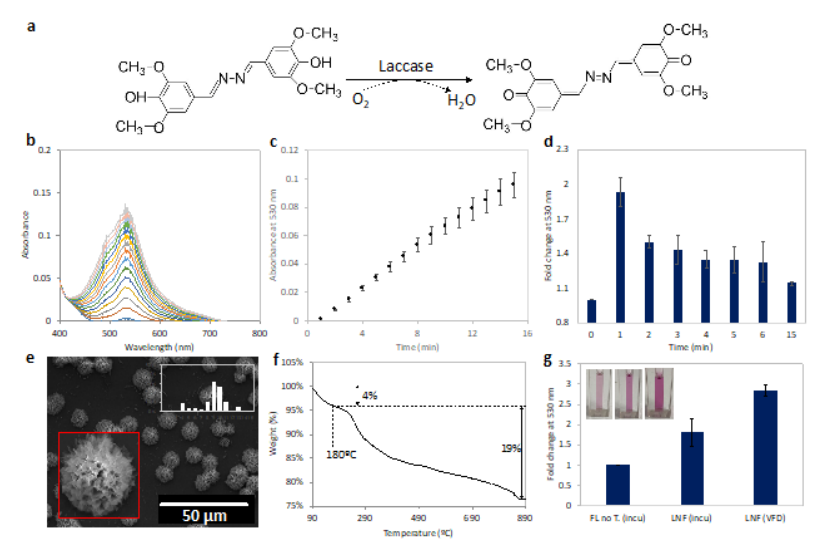

Figure 3: Oxidation of syringaldazine by free laccase and laccase NFs (LNFs). (a) Schematic of the laccase activity assay using syringaldazine as a substrate. The laccase converts syringaldazine into tetrametoxy azobismetrylene quinone. (b) UV-vis spectra of enzyme-substrate solutions of laccase at $0.625 \mathrm{mg} / \mathrm{mL}$ and 0.22 $\mathrm{mM}$ syringaldazine over $15 \mathrm{~min}$, recorded at $1 \mathrm{~min}$ intervals in a quartz cuvette. (c) Normalised changes in absorbance at $530 \mathrm{~nm}$ from (b) and plotted against the reaction time. (d) Fold acceleration of free laccase at different time points, determined by the ratio of the VFD-mediated substrate conversion to an identical laccase-syringaldazine solution (laccase at $0.625 \mathrm{mg} / \mathrm{mL}$ and syringaldazine at $0.22 \mathrm{mM}$ ) using incubation. (e) SEM of LNFs $\left(107.2 \mu \mathrm{L}\right.$ of $120 \mathrm{mM} \mathrm{CuSO}_{4}$ with $1 \mathrm{~mL}$ of $2.5 \mathrm{mg} / \mathrm{mL}$ laccase in $10 \mathrm{mM}$ PBS) and the size distribution plot from 60 randomly chosen LNFs. (f) STA profile of the as prepared LNFs. (g) Fold acceleration after 6 min reaction, determined by the ratio of the treated substrate conversion using FL (reaction conducted by incubation), LNFs formed using VFD (reaction by incubation), LNFs formed using VFD (reaction using the VFD at $5000 \mathrm{rpm}$ ) to an identical FL-syringaldazine solution (laccase at $1.702 \mathrm{mg} / \mathrm{mL}$ and syringaldazine at $0.22 \mathrm{mM}$ ) using incubation. FL: free laccase; incu: incubation.

Prominent SEM/EDX peaks on the timedependent samples were assigned to $\mathrm{Cu}, \mathrm{P}, \mathrm{O}, \mathrm{C}$ and $\mathrm{N}$, which is consistent with the hybrid flowers being mainly composed of $\mathrm{Cu}_{3}\left(\mathrm{PO}_{4}\right)_{2}$ and BSA (Figure S9). Elemental EDX maps on C, O, $\mathrm{P}$ and $\mathrm{Cu}$ overlap over the entire structure of BNFs. Over the time period of VFD processing examined (2 to $30 \mathrm{~min}$ ), the intensities for the elements increased, $\mathrm{C}$ to $1 \%, \mathrm{O} 1$ to $7 \%, \mathrm{Cu} 1$ to $4 \%$, and $\mathrm{P} 1$ to $3 \%$ In the absence of protein in the processing, micro-sized flowers $\sim 10 \mu \mathrm{m}$ in diameter form (Figure 2a). These results again highlight that the NF growth mechanism in the VFD is different from analogous BNFs prepared in previous studies (without VFD processing). Here they develop from nanoparticles to nanowires, to nanopetals and finally NFs. ${ }^{18}$ EDX maps for the particles formed in the present study show an efficient morphological transition from toroids to NFs with a homogeneous distribution of $\mathrm{Cu}$ and BSA. FT-IR analysis reveals peaks at 
$1540 \mathrm{~cm}^{-1}$ corresponding to -CONH stretching of BSA, and phosphate P-O vibrations at 1044 (asymmetric stretch) and $990 \mathrm{~cm}^{-1}$ (symmetric stretch). ${ }^{19}$ These peaks were also observed for BNFs (Figure 2b). XRD patterns show that the BNFs fit well with those for NFs without BSA incorporated and with $\mathrm{Cu}_{3}\left(\mathrm{PO}_{4}\right)_{2}$ from the database (Figure 2c). Taken together, the results establish that phosphate and BSA are both incorporated into the BNFs.

Previous studies have established that enzymatic reactions can be accelerated by pressure changes associated with Faraday waves contained within the thin films in VFD. ${ }^{20}$ In the present study, the VFD-mediated free laccase reaction achieved an average 54\% enhancement within 15 min, which shows a significant difference in fold change within the reaction time from $1 \mathrm{~min}$ to $15 \mathrm{~min}$ (Figure 3a-d). VFD processing facilities mass transfer and diffusion of oxygen ${ }^{21}$ which contributes to the $93 \%$ enhancement within the first minute of the reaction. The continuous reduction of the enhancement rate could be due to the concomitant reduction of dioxygen to water ${ }^{22}$ which potentially lowers the signal at $530 \mathrm{~nm}$ (Figure 3a). Compared to the optimised processing condition for BNFs $\left(6.7 \mu \mathrm{l}\right.$ of $120 \mathrm{mM} \mathrm{CuSO}_{4}$ with 1 $\mathrm{mL}$ of $0.1 \mathrm{mg} / \mathrm{mL}$ laccase at $5000 \mathrm{rpm}$ for 30 min), the same condition applied for LNFs showed a significant increase in the size of the NFs (from 2 to $4 \mu \mathrm{m}$ ) with non-compact petals (Figure S13), which arise from the low protein content of the as-received laccase $(\sim 1.2 \%)$. As a result, re-optimised conditions for preparing LNFs $(\sim 9 \mu \mathrm{m})$ using the VFD was required (Figure 3e-g, S14-16). The encapsulation efficiency was $61.3 \%$ (Table S1) and the weight percentage of encapsulated material in the corresponding NFs was 19\%, which might also contain impurities other than laccase (Figure 3f). Thermal gravimetric analysis, established a mass loss of $4 \%$ between 100 and $200{ }^{\circ} \mathrm{C}$ for LNFs, which corresponds to the loss of included water. The main decompositions with $19 \%$ mass loss occurred in higher temperatures arising from pyrolytic decomposition of the encapsulated material.

VFD-mediated acceleration of LNFs was compared to identical free laccase, and LNFs for activity assessment (Figure 3g). The activity of
LNFs exhibit $\approx 1.8$ fold increase compared to free laccase after both were incubated with syringadazine at room temperature for $6 \mathrm{~min}$. Further enhancement of LNFs activity $\approx 2.8$ fold was achieved by conducting the 6 min reaction in the VFD at $5000 \mathrm{rpm}\left(\theta=45^{\circ}\right)$. Close to accelerating free enzyme catalytic performance using VFD processing, ${ }^{23}$ the instantaneous pressure changes generated by Faraday waves and shear stress also increases LNFs catalysis in the VFD.

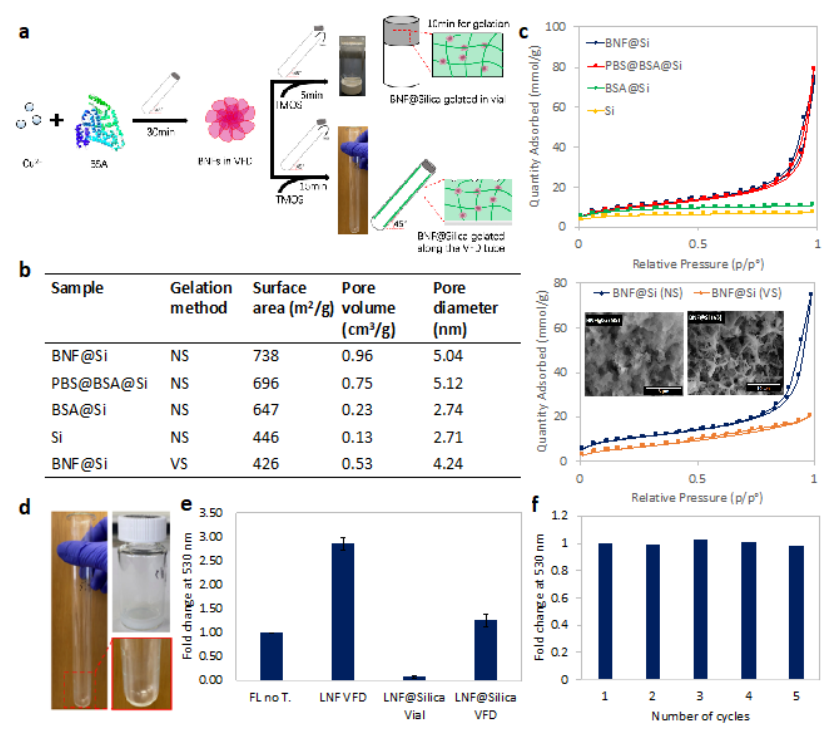

Figure 4. (a) Preparation of BNFs@Silica using the VFD compared to non-VFD. (b) Physisorption properties of silica hydrogels with different embedded materials prepared using different gelation methods as indicated. NS: naturally set; VS: VFD set, (c) Nitrogen adsorption analysis of BNFs@Silica and its composites prepared using different methods as indicated. Insets show corresponding SEM images. (d) Photographs of as-prepared VFD coupled LNF@Silica immobilization system (VLIS) and the glass vial coupled LNF@Silica immobilization system (GLIS). (e) Fold acceleration of LNF@Silica, determined by the ratio of the VFDmediated LNF, non-VFD mediated GLIS and VFD-mediated GLIS substrate conversion to an identical free laccasesyringaldazine solution (laccase at $1.702 \mathrm{mg} / \mathrm{mL}$ and syringaldazine at $0.22 \mathrm{mM}$ ) by incubation. (f) Reusability of VLIS.

As discussed, NFs are required to be separated by centrifugation for recycling use, but the integrity of the micron-sized structures, with fragile petals, can be destroyed during the multiple centrifugation processing. ${ }^{7}$ To overcome this problem we endeavoured to adhere/encapsulate LNFs to the surface of the tube using sol-gel chemistry. The as-prepared LNFs were mixed in silica hydrogel, which itself can be prepared in the VFD, ${ }^{16}$ and gelated along the VFD tube surface. The addition of PBS to the sol efficiently facilitates the complete gelation process down to 15 min while pro- 
cessing in the VFD at $5000 \mathrm{rpm}$ rotational speed. Using BSA as an optimising model (Figure 4a-c), nitrogen adsorption-desorption analyses showed variation in BET surface area and Barrett-JoynerHalenda $(\mathrm{BJH})$ pore volume of the resulting solgel, designated as BNFs@Silica (Figure 4b). The BET surface area of BNF@Silica incorporated in the VFD decreased to $426 \mathrm{~m}^{2} / \mathrm{g}$ with pore volume to $0.53 \mathrm{~cm}^{3} / \mathrm{g}$, compared to the sol-gel set without VFD processing. Addition of BNFs and PBS showed a slight shift of $\mathrm{Si}-\mathrm{O}-\mathrm{Si}, \mathrm{Si}-\mathrm{OH}$ and CONH vibrations in the FTIR (Figure S19). An intense Si-O-Si vibrational band is observed around $1063 \mathrm{~cm}^{-1}$, as expected for a dense silica network. The symmetric stretching vibration of Si-O-Si was observed at $800 \mathrm{~cm}^{-1}$, with the Si-O in-plane stretching vibration of silanol groups at around $945 \mathrm{~cm}^{-1} .{ }^{24}$ All samples were devoid of peaks representing - $\mathrm{CH}$ groups which would be present if there was incomplete hydrolysis and condensation of TMOS. ${ }^{16}$ To explore the catalytic performance of this VFD coupled LNF@Silica immobilization system or VLIS, $1 \mathrm{~mL}$ of syringaldazine was added to the VLIS followed by processing at $5000 \mathrm{rpm}$ rotational speed for 6 min. LNF@Silica incorporated in a glass vial or GLIS was used as the control (Figure 4d). VLIS showed a $\sim 16$ fold increase in syringadazine conversion compared to the control group of GLIS (Figure 4e). Two reasons could be responsible for this observation. Firstly, the high shear stress during the preparation of VLIS through the VFD minimises the build-up of alcohol during the initial hydrolysis process, which can eliminate the protein denaturation. Secondly, the acceleration of enzymatic activity could be ascribed to the increased substrate penetration while the tube is rotating, associated with shear stress double helical flow, as discussed above. Interestingly, the VFD coupled immobilized LNFs@Silica only shows a slight increase $(\approx 1.3$ fold) in catalytic activity when compared to free laccase. This is likely to be caused by the limited exposure of LNFs to the pressure waves associated with the Faraday waves in the VFD when immobilized within the silica gel. A similar situation has been observed when alkaline phosphatase displayed an average 6 fold acceleration in a VFD-mediated reaction, ${ }^{23}$ but once being immobilized on the glass surface, the VFD-mediated enzymatic ac- celeration is not observed. ${ }^{14,25}$ This results in high mass transfer (increase in average velocity of molecules in the system $)^{17}$ under conditions beyond diffusion control. For an enzyme like laccase, such conditions could accelerate removal of the product from the enzyme active site, decreasing product inhibition. The VLIS was tested for five consecutive cycles of use, being rinse three times with fresh PBS, and then reused again, without detectable loss of catalytic efficiency (Figure 4f).

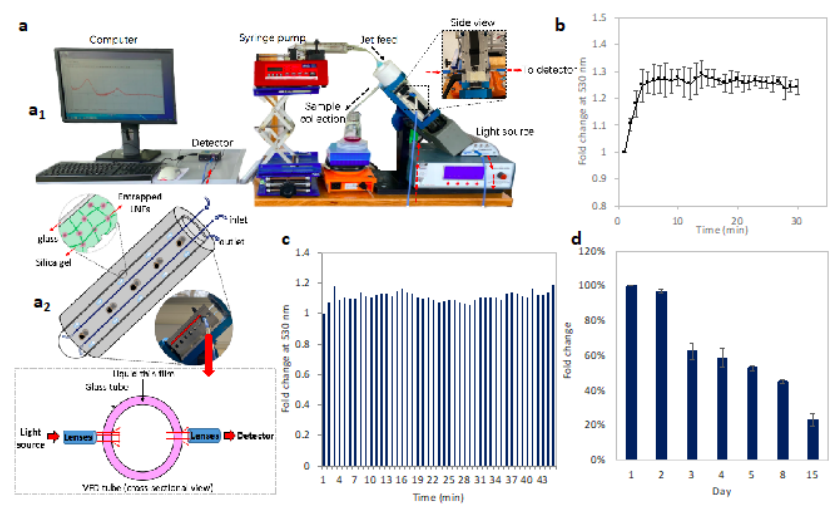

Figure 5. $\left(\mathrm{a}_{1}\right)$ Photograph of VLIS real-time monitoring system. (a2) Schematic of VLIS with optical spectroscopy for real-time monitoring. (b) Fold acceleration at $530 \mathrm{~nm}$ for $1 \mathrm{~mL}$ of syringaldazine $(0.22 \mathrm{mM})$ during $30 \mathrm{~min}$, recorded at 1 min intervals in a VLIS. (c) Real-time monitored reaction of $20 \mathrm{mM}$ syringadazine using VLIS at $5000 \mathrm{rpm}$ for $45 \mathrm{~min}$ under continuous flow at 0.4 $\mathrm{mL} / \mathrm{min}$. (d) Storing the VLIS at room temperature devoid of buffer resulted in $20 \%$ catalytic activity after 15 days.

To further explore the stability of this system, VLIS was tested against a continuous flow processing in real-time. After adding the substrate, the tube in the VFD was rotated at $5000 \mathrm{rpm}$ with a USB spectrophotometer (Ocean optics, USA) mounted around the tube, connected to fibre optics, using OceanView software (Figure 5a). As shown, the absorbance intensity increased in accordance with the processing time in the VFD, with the intensity almost plateaued after 4 min (Figure 5b). At a flow rate of $0.4 \mathrm{~mL} / \mathrm{min}$, the sample tube was able to maintain a stable level of syringadazine conversion for at least $45 \mathrm{~min}$, without laccase degradation (Figure $5 c$ ). The catalytic activity of a dried VLIS stored at room temperature showed a small change within the first $48 \mathrm{~h}$ after preparation (Figure $5 \mathrm{~d}$ ). The reduction of activity might be due to the usage of $11.6 \mathrm{mM} \mathrm{Cu}^{2+}$ during the LNF formation. Previous reports describe inhibition during the laccase 
oxidation reaction upon addition of $\mathrm{Cu}^{2+26,27}$ Having mentioned the low purity of laccase $(\sim 1.2 \%)$ in this study, $11.6 \mathrm{mM} \mathrm{Cu}^{2+}$ is used for LNF development and quantification purposes. Future research should focus on applying a purified laccase sample which could potentially lower the amount of $\mathrm{Cu}^{2+}$ required for LNF development.

\section{CONCLUSION}

The process developed herein uses a number of different applications of the VFD. The rate of fabrication of LNFs was dramatically reduced using VFD processing, albeit with a different mechanism of formation relative to conventional batch processing. Specifically, we observe a novel toroidal intermediate, which in itself is essentially a mould of the double helical driven topological fluid flow striking the surface of the tube in the VFD. LNFs accelerate the enzymatic reaction compared to free laccase, with further enhancement of LNFs activity achieved by conducting the reaction in the VFD. In addition, we have established that coating the VFD tube with LNF@Silica offers rapid UV-vis based monitoring of the enzymatic reaction, which has significantly enhanced catalytic activity ( 16 fold). Importantly, sample tubes have good reusability and stability in real-time processing, highlighting its potential as a microfluidic platform for practical applications. The utilisation of the VFD enables the generation and immobilization of LNFs into silica hydrogel, which greatly simplifies the fabrication process and shows potential in future biosensing research.

\section{ASSOCIATED CONTENT}

\section{Supporting Information}

Additional characterization data including SEM/EDX, UV-vis, Bio-Rad for protein quantification, STA and FTIR (PDF)

The Supporting Information is available free of charge on the ACS Publications website.

\section{AUTHOR INFORMATION}

\section{Corresponding Author}

*E-mail: xuan.luo@flinders.edu.au. Phone: +61 882012883. (X.L.)

*E-mail: colin.raston@flinders.edu.au. Phone: +61 882017958. Fax: +61 88201290 (C.L.R).

\section{Author Contributions}

The manuscript was written through contributions of all authors. / All authors have given approval to the final version of the manuscript.

\section{Funding Sources}

This work was supported by the Australia Research Council (DP 200101106) and the Government of South Australia, and the Australian Nanotechnology Network, through the Overseas Travel Fellowship program.

\section{Notes}

The authors declare the following competing financial interest(s): G.A.W. and C.L.R. have a financial interest in a company, Debut Biotechnology, which is commercializing some of the technologies described here. The terms of this arrangement have been reviewed and approved by the University of California in accordance with its conflict of interest policies.

\section{ACKNOWLEDGMENT}

The authors gratefully acknowledge the financial support from the Australia Research Council, the Government of South Australia, Australian Nanotechnology Network and the Australian Microscopy and Microanalysis Research Facility (AMMRF), the Australian National Fabrication Facility (ANFF) and the Flinders Microscopy for access to characterization facilities.

\section{ABBREVIATIONS}

VFD, Vortex fluidic device; NF, nanoflower; BSA, bovine serum albumin; BNF, BSA-incorporated nanoflower; LNF, laccaseincorporated nanoflower; VLIS, LNF@Silica incorporated in a VFD tube; GLIS, LNF@Silica incorporated in a glass vial.

\section{REFERENCES}

(1) Fernández-Fernández, M.; Sanromán, M. Á.; Moldes, D., Recent Developments and Applications of Immobilized Laccase. Biotechnol. Adv. 2013, 31, 1808-1825.

(2) Moldes, D.; Cadena, E. M.; Vidal, T., Biobleaching of Eucalypt Kraft Pulp with a Two Laccase-mediator Stages Sequence. Bioresource Technol. 2010, 101, 6924-6929.

(3) Rodríguez Couto, S.; Toca Herrera, J. L., Industrial and Biotechnological Applications of Laccases: A Review. Biotechnol. Adv. 2006, 24, 500-513.

(4) Ge, J.; Lei, J.; Zare, R. N., Protein-inorganic Hybrid Nanoflowers. Nat. Nanotechnol. 2012, 7, 428-432.

(5) Lee, S. W.; Cheon, S. A.; Kim, M. I.; Park, T. J., OrganicInorganic Hybrid Nanoflowers: Types, Characteristics, and Future Prospects. J. Nanobiotechnol. 2015, 13, 1-10.

(6) Sun, J.; Ge, J.; Liu, W.; Lan, M.; Zhang, H.; Wang, P.; Wang, Y.; Niu, Z., Multi-enzyme Co-embedded OrganicInorganic Hybrid Nanoflowers: Synthesis and Application as a Colorimetric Sensor. Nanoscale 2014, 6, 255-262.

(7) Ren, W.; Li, Y.; Wang, J.; Li, L.; Xu, L.; Wu, Y.; Wang, Y.; Fei, X.; Tian, J., Synthesis of Magnetic Nanoflower Immobilized Lipase and its Continuous Catalytic Application. New J. Chem. 2019, 43, 11082-11090.

(8) Kong, D.; Jin, R.; Zhao, X.; Li, H.; Yan, X.; Liu, F.; Sun, P.; Gao, Y.; Liang, X.; Lin, Y.; Lu, G., Protein-inorganic Hybrid Nanoflower-Rooted Agarose Hydrogel Platform for Point-of-Care Detection of Acetylcholine. ACS Appl. Mater. Inter. 2019, 11, 11857-11864.

(9) Dandoy, P.; Meunier, C. F.; Leroux, G.; Voisin, V.; Giordano, L.; Caron, N.; Michiels, C.; Su, B.-L., A Hybrid Assembly by Encapsulation of Human Cells within Mineralised Beads for Cell Therapy. PLOS ONE 2013, 8, 18.

(10) Mohidem, N. A.; Mat, H., Catalytic Activity and Stability of 
Laccase Entrapped in Sol-gel Silica with Additives. J. SolGel Sci. Techn. 2012, 61, 96-103.

(11) Mohidem, N. A.; Mat, H. B., The Catalytic Activity Enhancement and Biodegradation Potential of Free Laccase and Novel Sol-gel Laccase in Non-conventional Solvents. Bioresource Technol. 2012, 114, 472-477.

(12) Mohidem, N. A.; Mansor, A. F.; Zawawi. W.; Othman. N. S; Mat, H., Effect of Synthesis Conditions on Physical Properties, Laccase Catalytic Activity and Stability of SolGel Laccase. J. Sol-Gel Sci. Technol. 2016, 80, 587-597.

(13) Yuan, T. Z.; Ormonde, C. F. G.; Kudlacek, S. T.; Kunche, S.; Smith, J. N.; Brown, W. A.; Pugliese, K. M.; Olsen, T. J.; Iftikhar, M.; Raston, C. L.; Weiss, G. A., Shear-stressmediated Refolding of Proteins from Aggregates and Inclusion Bodies. ChemBioChem 2015, 16, 393-396.

(14) Britton, J.; Raston, C. L.; Weiss, G. A., Rapid Protein Immobilization for Thin Film Continuous Flow Biocatalysis. Chem. Commun. 2016, 52, 10159-10162.

(15) Luo, X.; Al-Antaki, A. H. M.; Harvey, D. P.; Ruan, Y.; He, S.; Zhang, W.; Raston, C. L., Vortex Fluidic Mediated Synthesis of Macroporous Bovine Serum Albumin-based Microspheres. ACS Appl. Mater. Inter. 2018, 10, 2722427232.

(16) Tong, C. L.; Stroeher, U. H.; Brown, M. H.; Raston, C. L., Continuous Flow Vortex Fluidic Synthesis of Silica Xerogel as a Delivery Vehicle for Curcumin. RSC Adv. 2015, 5, 79537958.

(17) Alharbi, T. M. D.; Jellicoe, M.; Luo, X.; Vimalanathan, K.; Alsulami, I. K.; Harbi, B. S. A.; Igder, A.; Chen, X.; Stubbs, K. A.; Chalker, J. M.; Zhang, W.; Boulos, R. A.; Jones, D. B.; Quinton, J. S.; Raston, C. L., Sub-micron Moulding Angled Vortex Fluid Flow. 2020 (under review)

(18) Kim, K. H.; Jeong, J.-M.; Lee, S. J.; Choi, B. G.; Lee, K. G., Protein-directed Assembly of Cobalt Phosphate Hybrid Nanoflowers. J. Colloid Interf. Sci. 2016, 484, 44-50.
(19) Yu, Y.; Fei, X.; Tian, J.; Xu, L.; Wang, X.; Wang, Y., Selfassembled Enzyme-inorganic Hybrid Nanoflowers and Their Application to Enzyme Purification. Colloid. Surface. B. 2015, 130, 299-304.

(20) Britton, J.; Meneghini, L.; Raston, C.; Weiss, G., Accelerating Enzymatic Catalysis Using Vortex Fluidics. Angew. Chem. Int. Edit. 2016, 55, 11387-11391.

(21) Pye, S. J.; Dalgarno, S. J.; Chalker, J. M.; Raston, C. L., Organic Oxidations Promoted in Vortex Driven Thin Films under Continuous Flow. Green Chem. 2018, 20, 118-124.

(22) Bento, I.; Silva, C. S.; Chen, Z.; Martins, L. O.; Lindley, P. F.; Soares, C. M., Mechanisms Underlying Dioxygen Reduction in Laccases. Structural and Modelling Studies Focusing on Proton Transfer. BMC Struct. Biol. 2010, 10, 114.

(23) Britton, J.; Meneghini, L. M.; Raston, C. L.; Weiss, G. A., Accelerating Enzymatic Catalysis Using Vortex Fluidics. Angew. Chem. Int. Edit. 2016, 55, 11387-11391.

(24) Al-Oweini, R.; El-Rassy, H., Synthesis and Characterization by FTIR Spectroscopy of Silica Aerogels Prepared Using Several $\mathrm{Si}(\mathrm{OR})_{4}$ and $\mathrm{R}^{\prime \prime} \mathrm{Si}\left(\mathrm{OR}^{\prime}\right)_{3}$ Precursors. J. Mol. Struct. 2009, 919, 140-145.

(25) Britton, J.; Dyer, R. P.; Majumdar, S.; Raston, C. L.; Weiss, G. A., Ten-Minute Protein Purification and Surface Tethering for Continuous-Flow Biocatalysis. Angew. Chem. Int. Edit. 2017, 56, 2296-2301.

(26) Lorenzo, M.; Moldes, D.; Rodríguez Couto, S.; Sanromán, M. A., Inhibition of Laccase Activity from Trametes versicolor by Heavy Metals and Organic Compounds. Chemosphere 2005, 60, 1124-1128.

(27) Capanni, C.; Taddei, N.; Gabrielli, S.; Messori, L.; Orioli, P.; Chiti, F.; Stefani, M.; Ramponi, G., Investigation of the Effects of Copper Ions on Protein Aggregation using a Model System. Cell. Mol. Life Sci. 2004, 61, 982-991. 


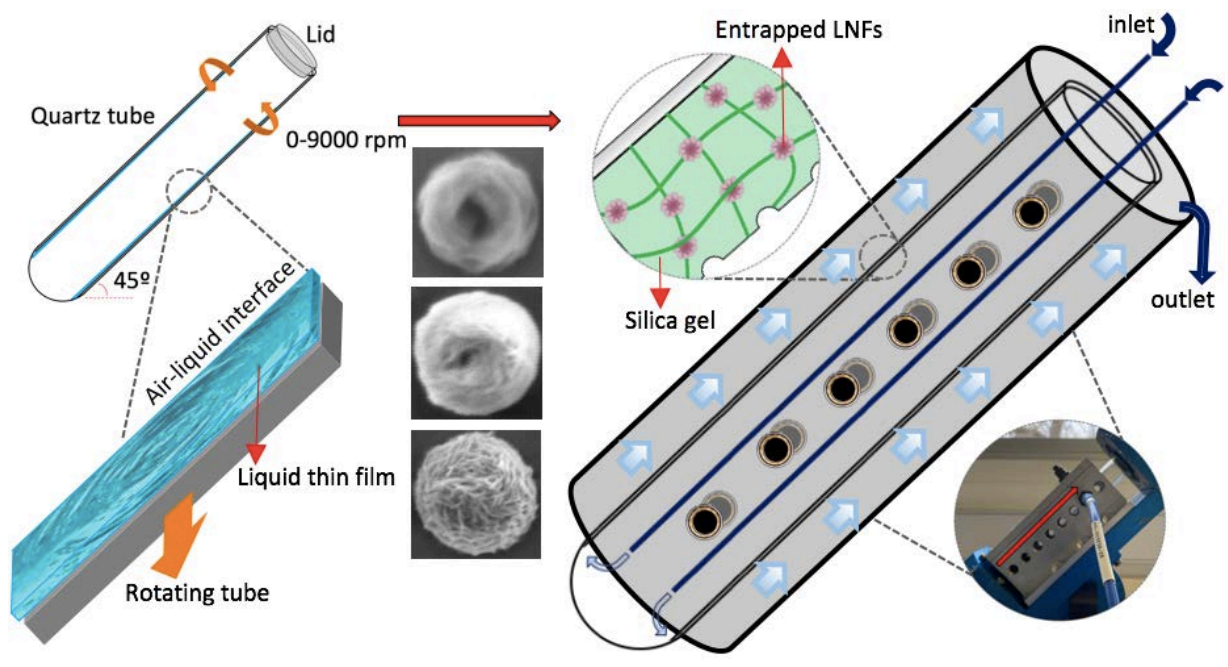

